\title{
Preparation of Magnetite Nanoparticles Coated with Silica via a Sol-gel Approach
}

\author{
Bing LIU, Deping WANG, Wenhai HUANG, Aihua YAO, Masanobu KAMITAKAHARA* and Koji IOKU* \\ School of Material Science and Engineering, Tongji University, 1239 Siping Load, Shanghai 200092, China \\ ${ }^{*}$ Graduate School of Environmental Studies, Tohoku University, 6-6-20, Aoba, Aramaki, Aoba-ku, Sendai 980-8579 Japan
}

\begin{abstract}
Silica-coated magnetite nanoparticles were synthesized via sol-gel process, which comprises the hydrolysis and condensation of TEOS in ethanol/water solution with $\mathrm{NH}_{3}$ aq. as catalyst. Transmission electron microscope and vibrating sample magnetometer was applied to characterize the nanocomposite particles. Results reveal that the reaction parameters including volume ratio of alcohol/water, the concentrations of $\mathrm{NH}_{3}$ aq and TEOS play important roles of the formation of silica shell. The thickness of silica shell increased with TEOS concentration. The nanocomposite particles remained favorable superparamagnetism. The formation of silica shell mainly came from the aggregative growth of silica primary particles on the surface of magnetite nanoparticles.
\end{abstract}

[Received August 29, 2007; Accepted October 18, 2007]

Key-words : Magnetite, Nanoparticle, Sol-gel

\section{Introduction}

Magnetite nanoparticles, due to their remarkable properties such as supeparamagnetism and high saturation maganetization, have received growing interest for their potential application in various fields, especially in biomedicine and bioengineering, such as targeted drug delivery, cell separation, magnetic resonance imaging, immunoassay and separation of biochemical products. ${ }^{1-3)}$ Most of these applications require the magnetic nanoparticles to be chemically stable, biocompatible and well dispersed in liquid media. However, as a result of anisotropic dipolar attraction, the unmodified magnetite nanoparticles tend to aggregate into large clusters, and thus lose the special magnetic properties associated with single-domain.

Surfactants are often used to create an electrostatic double layer to avoid the aggregation, however, the combination of surfactants with magnetite may not be firm enough and they may severely interfere with biomedical application. Usually, coating magnetite with an inert silica shell could help screen the magnetic dipolar attraction, which prevent the aggregation in liquid and improve their chemical stability. Meanwhile, abundant silanol groups are existed on the silica surface, allowing the easily attachment of functional groups by reacting with various coupling agents.

There are two major methods for the generation of silica shell on magnetic particles. The first method, known as reverse microemulsion synthesis, ${ }^{4)}$ relies on the use of large amount of surfactants to confine and control the coating of silica. Much effort is required to remove the surfactants. The second method is based on a sol-gel process, which comprises the hydrolysis and condensation of silicon alkoxides in a basic ethanol/water solution. This method has been applied to coat magnetic particles, ${ }^{5)-7)}$ however, few works have been directed to study the effects of the reactants on formation of welldefined silica-coated magnetic particles and the formation mechanism of silica shell. In this paper, we demonstrated the preparation of ultra-stable colloidal magnetite nanoparticles, and systematically studied the effects of the volume ratio of ethanol/water, $\mathrm{NH}_{3}$ aq. and TEOS concentrations on silica shell. Finally, a formation mechanism of silica shell was proposed.

\section{Experimental}

2.1 Preparation of colloidal magnetite nanoparticles

$\mathrm{FeCl}_{3} \cdot 6 \mathrm{H}_{2} \mathrm{O}$ and $\mathrm{FeCl}_{2} \cdot 4 \mathrm{H}_{2} \mathrm{O}$ were purchased from China
Medicine (Group) Shanghai Chemical Reagent Corporation. $\mathrm{NH}_{3}$ aq. (25 mass\% aqueous solution), ethanol and tetraethyl orthosilicate (TEOS) were obtained from Sinopharm Chemical Reagent Co., Ltd.

Magnetite nanoparticles were prepared using the method already described based on the chemical coprecipitation of $\mathrm{Fe}^{2+}$ and $\mathrm{Fe}^{3+}$ by adding a concentrated $\mathrm{NH}_{3}$ aq. (25 mass \%). ${ }^{8} \quad 0.5 \mathrm{~g}$ magnetite nanoparticles were dispersed in $100 \mathrm{~cm}^{3}$ citric acid solution $(0.05 \mathrm{M})$ under magnetic stirring, and the $\mathrm{pH}$ was adjusted to 5.5 by $\mathrm{NH}_{3}$ aq. ( 25 mass\%). After $4 \mathrm{~h}$ the magnetite nanoparticles were precipitated by acetone and washed with acetone by magnetic decantation to remove the redundant citric acid. Subsequently, $100 \mathrm{~cm}^{3}$ water was added to redisperse the ultrafine magnetic particles.

2.2 Silica coating to magnetite nanoparticles

Magnetite nanoparticles were coated with silica at room temperature in a basic ethanol/water solution by a sol-gel approach which was also known as the Stöber method. First, $2 \mathrm{~cm}^{3}$ of the magnetite solution was diluted with $80 \mathrm{~cm}^{3}$ ethanol, appropriate amount of water and appropriate amount of $\mathrm{NH}_{3}$ aq. $(25$ mass $\%$ ). Then the mixture was homogenized by ultrasonicatic vibration. Finally, under continuous stirring, TEOS was added slowly to the solution. The reaction, involving the hydrolysis of TEOS and subsequent condensation of silica onto the surfaces of magnetite cores, was allowed to proceed for $8 \mathrm{~h}$. The final core-shell nanoparticles was separated by centrifuging and washed with water 4 times.

\subsection{Characterization}

The produced phases were indentified by a powder X-ray diffractometer with graphite-monochromatized $\mathrm{CuK} \alpha$ radiation, operated at $40 \mathrm{kV}$ and $100 \mathrm{~mA}$ (XRD, RIGAKU, D/ max2550, JAPAN). The crystallite size was calculated by Scherrer's formula. The size and morphology of the core-shell nanoparticles were characterized by transmission electronic microscopy (TEM, HITACHI H-800, $200 \mathrm{kV}$ ). Magnetic properties of the samples were characterized by vibrating sample magnetometer (VSM, Nanjing University Instrument Plant LH-3).

\section{Result and discussion}

3.1 Effect of volume ratio of ethanol/water

The diameter of magnetite nanoparticles observed by TEM was almost the same as their crystallite size estimated by XRD using Scherrer's formula of about $12 \mathrm{~nm}$. Figure 1 shows TEM images of magnetite nanoparticles coated with silica 

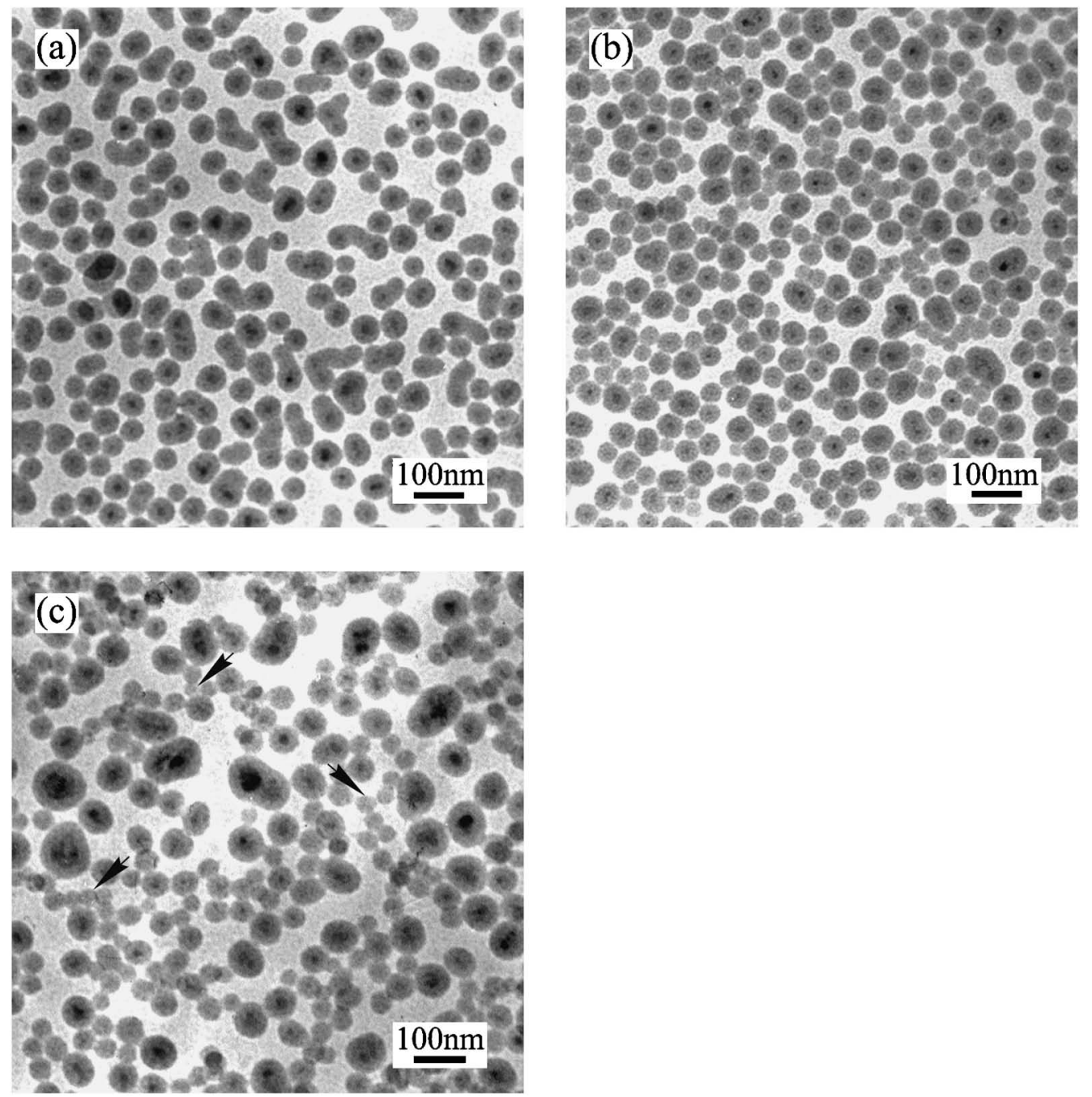

Fig. 1. TEM images of $\mathrm{SiO}_{2} / \mathrm{Fe}_{3} \mathrm{O}_{4}$ nanocomposite particles prepared under different volume ratio of ethanol/water (a: $8: 1, \mathrm{~b}: 4: 1$, c: $\left.2: 1\right)$.

$\left(\mathrm{SiO}_{2} / \mathrm{Fe}_{3} \mathrm{O}_{4}\right)$ prepared under different volume ratio of ethanol/water $\left(V_{\mathrm{E} / \mathrm{M}}\right)$ values while the amounts of other reagents were the same: $0.4 \mathrm{M} \mathrm{NH}_{3}$ aq. and $0.02 \mathrm{M}$ TEOS. $\mathrm{SiO}_{2} / \mathrm{Fe}_{3} \mathrm{O}_{4}$ nanoparticles could be formed in a large range of $V_{\mathrm{E} / \mathrm{w}}$ as shown in Fig. 1 (a) - (c), however, the morphology of the particles was different. A lot of irregular particles were generated at a high $V_{\mathrm{E} / \mathrm{M}}$ value of $8: 1$ (Fig. 1(a)), with the decrease of $V_{\mathrm{E} / \mathrm{M}}$ value to $4: 1$, the particles became more spherical and uniform (Fig. 1(b)), while the $V_{\mathrm{E} / \mathrm{M}}$ value further decreased to $2: 1$, many magnetite-free silica particles (noted by arrows) and irregular $\mathrm{SiO}_{2} / \mathrm{Fe}_{3} \mathrm{O}_{4}$ nanoparticles with cores of larger clusters were formed.

The polarity of the reaction media, which has effect on the surface charge of colloidal particles increased upon the decrease of $V_{\mathrm{E} / \mathrm{M}}$ value. ${ }^{9)}$ When at a high $V_{\mathrm{E} / \mathrm{M}}$ value, the low polarity of the media would result in the decrease of surface charge of colloidal magnetite and silica shell which was generated at the early stage. As a result, the electrostatic repulsion between particles would be reduced, leading to the aggregation of magnetite cores and irregular morphology of the final products. With the decrease of $V_{\mathrm{E} / \mathrm{M}}$ value, the colloidal particles become better dispersed, and it's more favorable for preparation of more regular core-shell particles with little clusters, as shown in Fig. 1(b). However, when the $V_{\mathrm{E} / \mathrm{M}}$ value decreased further, the hydrolysis and condensation of
TEOS would become over rapid as a result of large amount of water, which may lead to the formation of lots of silica primary particles in the media. These primary particles couldn't be completely captured by magnetite nanoparticles and some of them may aggregate together to form stable secondary silica spheres (noted by arrows in Fig. 1(c)) with a typical aggregative growth mechanism. ${ }^{10)}$ Furthermore, the increase in water volume ratio promotes the dissociation of $\mathrm{NH}_{3}$ aq. and brings about an increase in ionic strength, leading to the reduction in the thickness of electrostatic double layer, and then magnetite particles become unstable and tend to aggregate into clusters. As a result, some large irregular particles with cores of magnetic clusters are founded.

\subsection{Effect of $\mathrm{NH}_{3}$ aq. concentration}

Figure 2 shows TEM images of $\mathrm{SiO}_{2} / \mathrm{Fe}_{3} \mathrm{O}_{4}$ nanoparticles prepared under different $\mathrm{NH}_{3}$ aq. concentrations while the amounts of other reagents with the same $V_{\mathrm{E} / \mathrm{M}}$ value of $4: 1$ and $0.02 \mathrm{M}$ TEOS. No core-shell $\mathrm{SiO}_{2} / \mathrm{Fe}_{3} \mathrm{O}_{4}$ nanoparticles or $\mathrm{SiO}_{2}$ nanospheres formed under $0.15 \mathrm{M} \mathrm{NH}_{3}$ aq. as shown in Fig. 2 (a) while irregular core-shell nanoparticles with cluster cores were generated under a high $\mathrm{NH}_{3}$ aq. concentration as shown in Fig. 2(b).

To the base-catalyzed hydrolysis and condensation of TEOS, the surface potential and ionic strength are considered as the main factors controlling the silica growth. ${ }^{11)-13)}$ The 

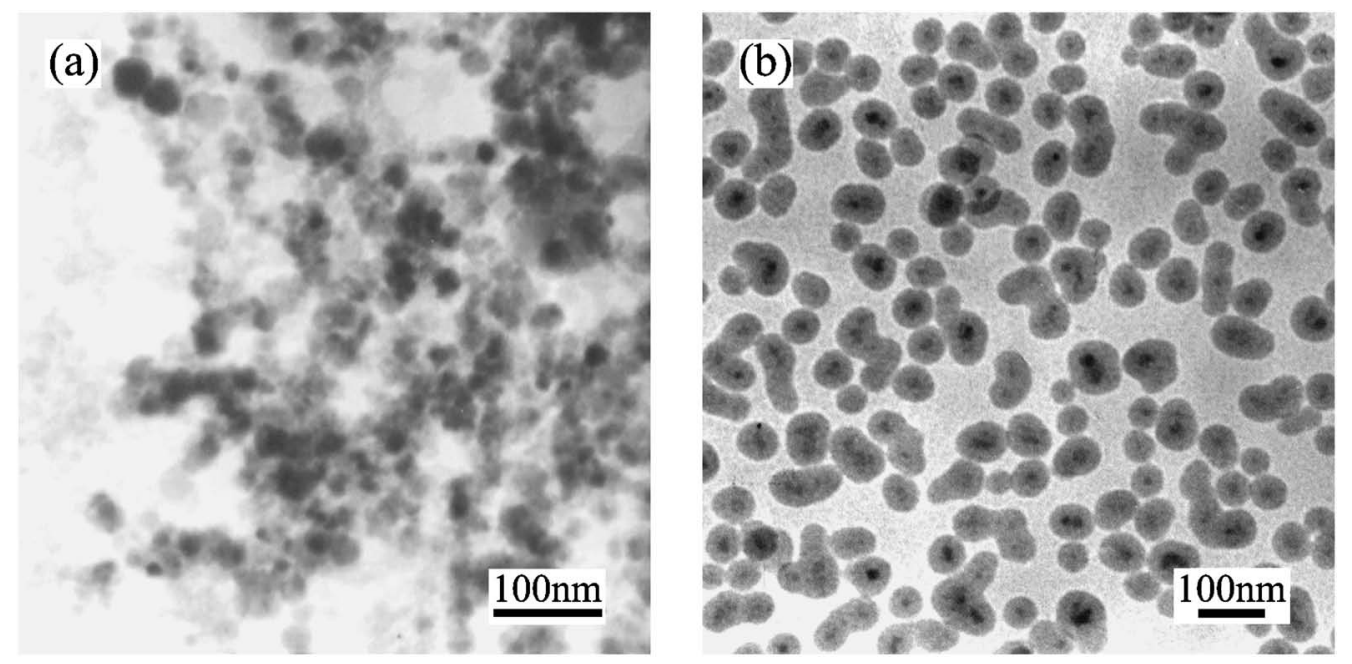

Fig. 2. TEM images of $\mathrm{SiO}_{2} / \mathrm{Fe}_{3} \mathrm{O}_{4}$ nanocomposite particles prepared under different $\mathrm{NH}_{3}$ aq. concentrations (a: $0.15 \mathrm{M}$, b: $0.6 \mathrm{M}$ ).
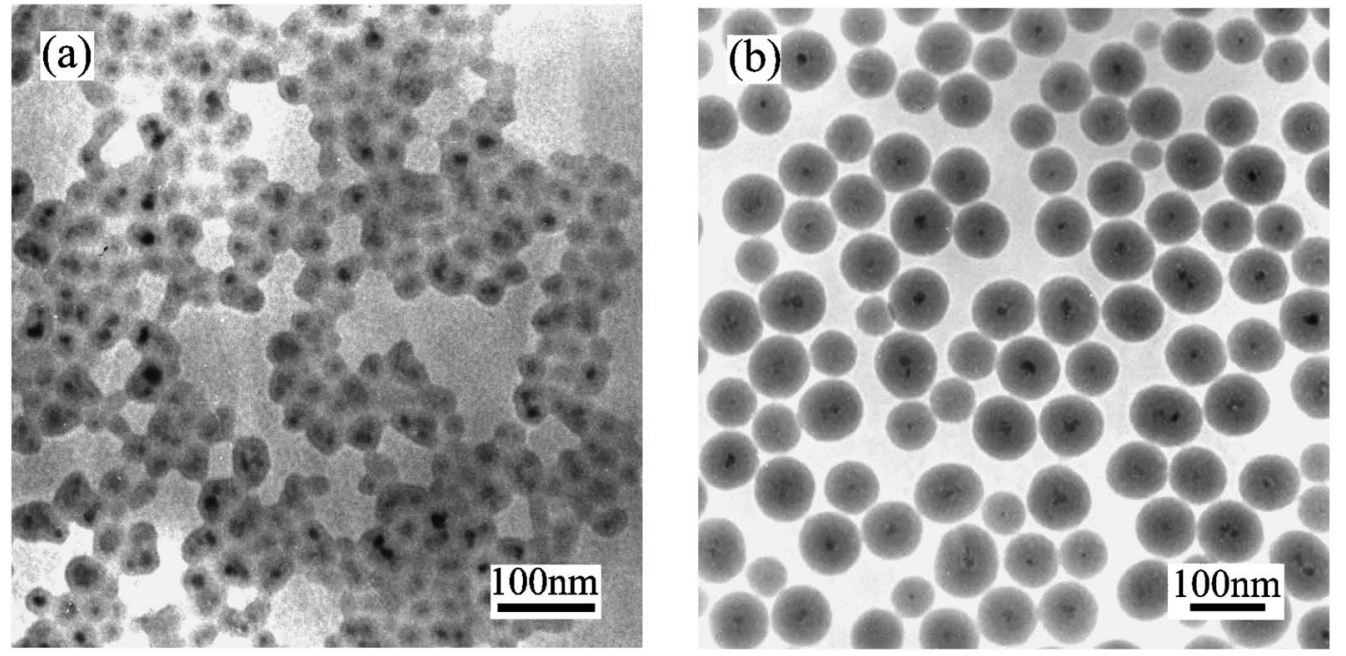

Fig. 3. TEM images of $\mathrm{SiO}_{2} / \mathrm{Fe}_{3} \mathrm{O}_{4}$ nanocomposite particles prepared under different TEOS concentrations (a: $0.01 \mathrm{M}$, b: $0.05 \mathrm{M}$ ).

stability of colloids is the coaction of van der Waals attraction and electrostatic repulsion. As increasing the ionic strength, more reverse charged ions would enter into the electrostatic double layer and decrease the colloidal surface potential, leading to reduction of electrostatic repulsion and then the particles incline to aggregate.

Silica primary particles are formed almost immediately when the TEOS was added into the basic ethanol/water solution by the condensation of $\mathrm{Si}\left(\mathrm{OC}_{2} \mathrm{H}_{5}\right)_{4-n} \mathrm{O}_{n}{ }^{n-}$ which is generated by the partial hydrolysis of TEOS. ${ }^{14)}$ When at a low $\mathrm{NH}_{3}$ aq. concentration, the ionic strength of the media becomes low and the surface potential of particles is high, resulting in the strong electrostatic repulsion between particles. Consequently, the silica primary particles are hard to collide with magnetite and aggregate to form a shell on the surface. Meanwhile, silica primary particles also couldn't aggregate to form secondary silica nanospheres via the aggregative growth as a result of strong electrostatic repulsion. Thus, the core-shell $\mathrm{SiO}_{2} / \mathrm{Fe}_{3} \mathrm{O}_{4}$ nanoparticles or silica nanospheres weren't formed in Fig. 2 (a).

The ionic strength of the media increased with $\mathrm{NH}_{3}$ aq. concentration, and correspondingly, the surface potential of particles was reduced. Most of silica primary particles were captured by magnetite and the generation of secondary silica spheres was suppressed. Core-shell $\mathrm{SiO}_{2} / \mathrm{Fe}_{3} \mathrm{O}_{4}$ nanoparticles were formed as shown in Fig. 1(b) under $0.4 \mathrm{M} \mathrm{NH}_{3}$ aq.. ${ }^{12)}$ However, when the $\mathrm{NH}_{3}$ aq. concentration was further increased to $0.6 \mathrm{M}$, many irregular $\mathrm{SiO}_{2} / \mathrm{Fe}_{3} \mathrm{O}_{4}$ nanoparticles were generated as shown in Fig. 2(b). The high ionic strength caused the dramatic decrease in surface potential of colloidal particles and subsequently, some of the magnetite particles probably aggregated into clusters before silica coating and silica shell formed at the early stage tended to aggregate into large $\mathrm{SiO}_{2} / \mathrm{Fe}_{3} \mathrm{O}_{4}$ clusters with ill morphology.

\subsection{Effect of TEOS concentration}

Figure 3 shows the TEM images of $\mathrm{SiO}_{2} / \mathrm{Fe}_{3} \mathrm{O}_{4}$ nanoparticles prepared under different TEOS concentrations while the amounts of other reagents were the same: $4: 1 V_{\mathrm{E} / \mathrm{M}}$ value and $0.4 \mathrm{M} \mathrm{NH}_{3}$ aq.. Together with Fig. 1(b) and Fig. 3 reveals that the thickness of silica shell can be controlled by TEOS concentrations. With the increase of TEOS concentration, the shell thickness shown in Figs. 3(a), 1(b) and 3(b) are about 10,20 and $36 \mathrm{~nm}$ respectively, furthermore, the particles become more uniform and spherical. 


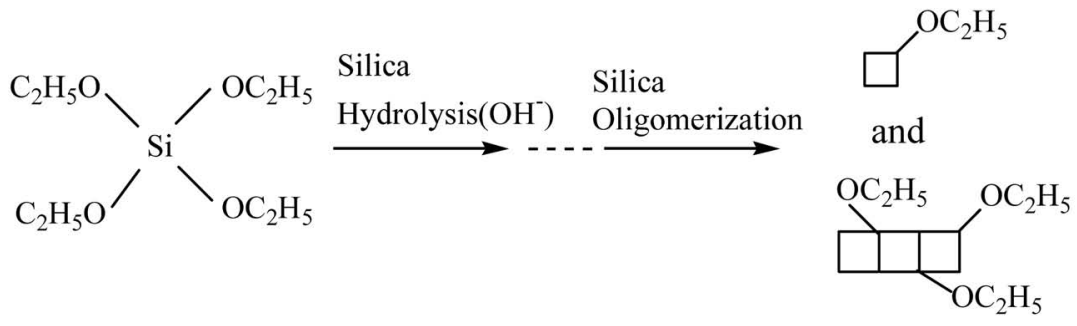

(D4R)

(TEOS)

(Monomeric Silica)

(Silica Primary Particles)<smiles>CCOC1CC2(OCC)C(OCCOC3CCC3)C1C1(OCC)CCC21</smiles>

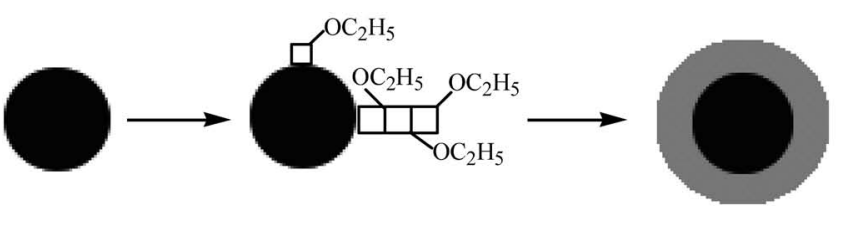
(Silica Primary
$\left(\mathrm{Fe}_{3} \mathrm{O}_{4}\right)$
(Intermediate)
$\left(\mathrm{SiO}_{2} / \mathrm{Fe}_{3} \mathrm{O}_{4}\right)$

Fig. 4. Schematic illustration of the mechanism for the formation of silica shell on the surface of magnetite particles.

\subsection{Formation mechanism of silica shell}

Based on the work presented previously, ${ }^{10)-15)}$ we propose that the silica shell forms via two main stages:

The first stage involves the hydrolysis and condensation of TEOS. Monomeric silica, in the form of $\mathrm{Si}\left(\mathrm{OC}_{2} \mathrm{H}_{5}\right)_{4-n} \mathrm{O}_{n}{ }^{n-}$, is generated by partial hydrolysis of TEOS. TEOS concentration used in this work is much higher than the approximate saturation limit of monomeric silica $(0.0002 \mathrm{M})$, therefore, considerable silica primary particles are formed immediately upon the addition of TEOS by the condensation of monomeric silica. ${ }^{14)}$ According to the work of Nooney and co-workers, the primary particles might be mainly in the form of D4R (double four-ring) and $n \mathrm{D} 4 \mathrm{R} .{ }^{14}$ )

The second stage, growth of silica on the magnetite surface, may proceed via two routes: (1) the aggregative growth of silica primary particles on the magnetite seeds; (2) deposition of monomeric silica on the seeds. It's conceivable that the growth of silica shell can proceeds via route (2) because of the high reactivity of monomeric silica. However, considerable silica primary particles exist in this basic ethanol/water solution, and thus the growth most probably proceeds via route (1). Silica primary particles colloid and aggregate with magnetite, then the primary particles consecutively aggregate on the seeds surface to form the shell. The schematic illustration of the mechanism for the formation of silica shell on the surface of magnetite particles is shown in Fig. 4.

\subsection{The magnetic properties}

The hysteresis loops of magnetite and $\mathrm{SiO}_{2} / \mathrm{Fe}_{3} \mathrm{O}_{4}$ nanoparticles with different thickness of silica shell were measured at room temperature using a vibrating sample magnetometer. As could be seen in Fig. 5, the magnetite nanoparticles show favorable superparamagnetic behaviors and saturation magnetization $\left(M_{\mathrm{s}}\right)$ of $64.5 \mathrm{~A} \cdot \mathrm{m}^{2} / \mathrm{kg}$. Similar with the magnetite, $\mathrm{SiO}_{2} / \mathrm{Fe}_{3} \mathrm{O}_{4}$ nanoparticles show superparamagnetic property, indicating that the silica shell almost has no effect on the coercive force. In addition, the $\mathrm{Ms}$ of $\mathrm{SiO}_{2} / \mathrm{Fe}_{3} \mathrm{O}_{4}$ nanoparticles decrease correspondingly upon the increase of the thickness of silica shell, which is resulted from the decrease of mass proportion of magnetite.

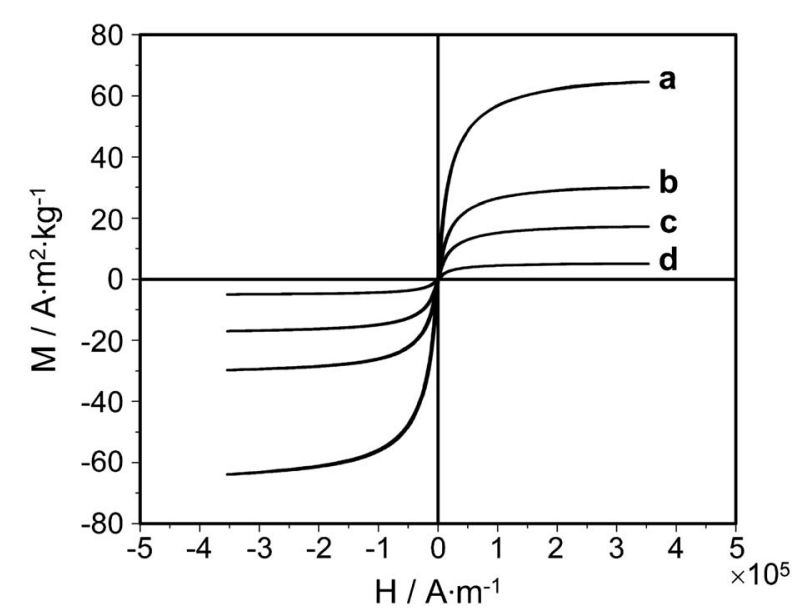

Fig. 5. Hysteresis loops of $\mathrm{Fe}_{3} \mathrm{O}_{4}$ (a) and $\mathrm{SiO}_{2} / \mathrm{Fe}_{3} \mathrm{O}_{4}$ nanoparticles with $10(\mathrm{~b}), 20(\mathrm{c})$ and $36 \mathrm{~nm}$ shell (d).

\section{Conclusion}

The formation of silica shell on magnetite nanoparticles via a sol-gel approach was systematically studied. The results showed that $V_{\mathrm{E} / \mathrm{M}}$, concentrations of $\mathrm{NH}_{3}$ aq. and TEOS had effects on the formation of silica shell. At high $V_{\mathrm{E} / \mathrm{M}}$, irregular $\mathrm{SiO}_{2} / \mathrm{Fe}_{3} \mathrm{O}_{4}$ nanoparticles were obtained while many secondary silica nanospheres were produced at low $V_{\mathrm{E} / \mathrm{M}} \cdot \mathrm{NH}_{3}$ aq. concentration played an important role on the formation of silica shell. As increasing TEOS concentration, the silica shell increased, $\mathrm{SiO}_{2} / \mathrm{Fe}_{3} \mathrm{O}_{4}$ nanoparticles became more regular and spherical, and the $M_{\mathrm{s}}$ decreased correspondingly. In addition, all the particles exhibited superparamagnetic propeties. The formation of silica shell can probably explained by the aggregative growth of silica primary particles on the seeds surface.

Acknowledgements This work was supported by the National Natural Science Foundation of China (NSFC) (No. 50572072) and Special Founds from Science and Technology Commission of Shanghai Municipality (SCTSM) (No. 0452nm059). 


\section{References}

1) J. Kim, J. E. Lee, J. Lee, J. H. Yu, B. C. Kim, K. An, Y. Hwang, C. Shin, J. G. Park, J. Kim and T. Hyeon, J. Am. Chem. Soc., 128, 688-689 (2006).

2) M. Arruebo, M. Galán, N. Navascués, C. Téllez, C. Marquina, M. R. Ibarra and J. Santamaría, Chem. Mater., 18, 1911-1919 (2006).

3) H. Zhang and M. E. Meyerhoff, Anal. Chem., 78, 609-616 (2006).

4) D. K. Yi, S. S. Lee, G. C. Papaefthymiou and J. Y. Ying, Chem. Mater., 18, 614-619 (2006).

5) Y. Lu, Y. Yin, B. T. Mayers and Y. Xia, Nano Lett., 2, 183-186 (2002).

6) A. P. Philipse, M. P. B. Bruggen and C. Pathmamanoharan, Langmuir, 10, 92-99 (1994).

7) X. Liu, Z. Ma, J. Xing and H. Liu, J. Magn. Magn. Mater., 270, 1-6 (2004).
8) M. Lou, D. Wang, W. Huang, D. Chen and B. Liu, J. Magn. Magn. Mater., 305, 83-90 (2006).

9) Y. H. Deng, C. C. Wang, J. H. Hu, W. L. Yang and S. K. Fu, Colloids Surf., A: Phys. Eng Asp., 262, 87-93 (2005).

10) G. H. Bogush and C. F. Zukoski IV, J. Colloid Interface Sci., 142, 19-34 (1991).

11) D. Nagao, T. Satoh and M. Konno, J. Colloid Interface Sci., 232, 102-110 (2000).

12) D. Nagao, Y. Kon, T. Satoh and M. Konno, J. Chem. Eng. Jpn., 33, 468-473 (2000).

13) A. V. Blaaderen, J. V. Geest and A. Vrij, J. Colloid Interface Sci., 154, 481-501 (1992).

14) R. I. Nooney, D. Thirunavukkarasu, Y. Chen, R. Josephs and A. E. Ostafin, Langmuir, 19, 7628-7637 (2003).

15) E. Mine, A. Yamada, Y. Kobayashi, M. Konno and L. M. Liz-Marzán, J. Colloid Interface Sci., 264, 385-390 (2003). 